\title{
Efficacy and Safety of Octagam ® in Patients With Chronic Inflammatory Demyelinating Polyneuropathy
}

\author{
Chafké Belmokhtar · Pierre Lozeron · David Adams · Jérôme Franques · \\ Arnaud Lacour - Etienne Godet - Mathieu Bataille - Odile Dubourg · \\ Gilles Angibaud · Emilien Delmont · Françoise Bouhour • \\ Philippe Corcia $\cdot$ Jean Pouget
}

Received: January 10, 2019 / Published online: March 22, 2019

(C) The Author(s) 2019

\section{ABSTRACT}

Introduction: Chronic inflammatory demyelinating polyradiculoneuropathy (CIDP) is a debilitating autoimmune neuropathy that is treated with intravenous immunoglobulin (IVIG). The aim of this retrospective study was

Enhanced Digital Features To view enhanced digital features for this article go to https://doi.org/10.6084/m9. figshare.7836449.

C. Belmokhtar $(\bowtie)$

Octapharma SAS, 62 bis Avenue André Morizet, Boulogne-Billancourt, 92100 Paris, France

e-mail: chafke.belmokhtar@octapharma.com

P. Lozeron

Lariboisiere University Hospital, 2 Rue Ambroise

Paré, 75010 Paris, France

D. Adams

INSERM UMR115 and Kremlin Bicetre University Hospital, Assistance Publique-Hôpitaux de Paris (APHP), 78 Rue du Général Leclerc, 94270 Le Kremlin-Bicêtre, France

J. Franques $\cdot$ J. Pouget

La Timone University Hospital,, Assistance

Publique-Hôpitaux de Marseilles (APHM), 264 rue

Saint Pierre, 13005 Marseille, France

A. Lacour

Lille University Hospital, Avenue Oscar Lambret, 59000 Lille, France

E. Godet

Bon-Secours Hospital, 1 Place Philippe de Vigneulles, 57000 Metz, France to investigate the efficacy and safety of the sucrose-free IVIG Octagam ${ }^{\circledR}$ (Octapharma AG, Lachen, Switzerland) in patients with CIDP.

Methods: Data from 47 patients who received at least one dose of Octagam were collected from the records of 11 centres in France. Efficacy was assessed using Overall Neuropathy Limitation Scale (ONLS). Safety was evaluated using adverse event rates.

M. Bataille

Caen University Hospital, Avenue de La Côte de Nacre, 14003 Caen, France

O. Dubourg

Pitié-Salpêtrière University Hospital, 47-83

Boulevard de l'Hôpital, 75013 Paris, France

G. Angibaud

Pont de Chaume Clinic, 330 Avenue Marcel Unal, 82000 Montauban, France

E. Delmont

Nice University Hospital, 4 Avenue Reine Victoria, 06003 Nice Cedex 1, France

F. Bouhour

Pierre Wertheimer Hospital, 59 Boulevard Pinel, 69677 Lyon-Bron, France

P. Corcia

Tours University Hospital, 2 Boulevard Tonnellé, 37000 Tours, France 
Results: Data from 24 patients who were IVIG naïve $(n=11)$ or had stopped IVIG $\geq 12$ weeks before initiation of Octagam therapy (washout group; $n=13$ ) were included in the efficacy analysis. At 4 months post-initiation of Octagam treatment, $41.7 \%$ of patients had improved their functional status (decrease of $\geq 1$ ONLS score) with a significant change in the ONLS score from baseline $(-0.42 ; p=0.04$; signed test). Functional status was reduced in only two patients: one patient in the IVIG-naïve group and one patient in the IVIG-washout group. All 47 patients were included in the safety analysis, which showed that Octagam was well tolerated, with a frequency of 0.04 adverse events per Octagam course. The most common adverse drug reaction was headache.

Conclusions: These real-life results are consistent with the efficacy and safety of IVIG reported in randomised controlled studies. A longterm prospective study of Octagam in patients with CIDP is warranted.

Funding: Octapharma, France SAS.

Keywords: Chronic inflammatory demyelinating polyneuropathy; Immunoglobulins; Immunotherapy; Octagam ${ }^{\circledR} ; \quad$ Peripheral nervous system disease

\section{INTRODUCTION}

Chronic inflammatory demyelinating polyradiculoneuropathy (CIDP) is an uncommon autoimmune polyneuropathy with heterogeneous presentation and prognosis [1]. The prevalence of definite and probable CIDP is estimated at 1-7.7 cases per 100,000 adults [2-5]. While CIDP can occur at any age, the prevalence increases with age, peaking in the seventh decade [3].

CIDP runs a progressive course of remission and relapse, leading to a pronounced disability over a period of several months [6]. The typical presentation includes symmetric, distal and proximal weakness with areflexia and sensory loss. Electrophysiological features include mainly reduced nerve conduction velocities, abnormal temporal dispersion and conduction blocks [7-9]. Cerebrospinal fluid examination often shows isolated high protein levels [6]. The heterogeneous nature of CIDP's immune pathology is becoming better understood. While the classical pathology is macrophagemediated demyelination, studies have identified a subset of patients in whom there is paranodal dissection (detachment of myelin terminal loops from the axolemma [10]) without macrophage-mediated demyelination [11]; instead, these patients have autoantibodies to paranodal junction proteins, such as neurofascin-155 and contactin-1 [11].

First-line treatments for CIDP include corticosteroids, intravenous immunoglobulin (IVIG) and plasma exchange (PE) [12-15]. The shortterm benefit of IVIG is well documented, with studies demonstrating IVIG efficacy up to 6 weeks $[8,9,16-21]$; however, the long-term benefits of IVIG and how maintenance treatment should be given are still mostly unknown [22]. One prospective, placebo-controlled, crossover trial assessed IVIG in patients for up to 48 weeks (ICE trial) [23]. An open-label study in Japan investigating the efficacy and safety of long-term IVIG therapy showed an even higher responder rate at 28 weeks than in the ICE trial and also a low relapse rate at 52 weeks [24]. Another retrospective study included only patients with at least 48 weeks of follow-up [25]. Both of these retrospective studies demonstrated similar results and a beneficial effect of IVIG also after long-term treatment. In head-to-head comparisons, no differences in short-term efficacy between IVIG and PE or corticosteroids have been demonstrated $[26,27]$. However, given the potent adverse events (AEs) associated with long-term corticosteroid use and the cost of and limited access to PE, IVIG seems to be a suitable first-choice therapy [28], as it is well tolerated and less frequently discontinued due to inefficacy, AEs or intolerance than treatment with 6-month intravenous methylprednisolone [29].

Recent evidence suggests that therapeutic response to IVIG may depend on the underlying pathology. Patients in whom autoantibodies to paranodal junctional proteins have been detected may be refractory to IVIG [10]. In contrast, those with typical CIDP, and thus classical macrophage-induced demyelination, respond to treatment with IVIG [10]. Further research is 
Table 1 Patient demographics and exposure to Octagam treatment in the efficacy analysis population

Patient demographics and exposure to Octagam treatment

Values $(n=24$ patients)

Gender, $n(\%)$

Male

Female

Age at enrolment (years)

$62.8 \pm 11.0[46-79]$

Age at disease diagnosis (years)

$59.2 \pm 14.9[22-79]$

Disease onset (months)

$52.1 \pm 92.3[0-346]$

Baseline ONLS

$3.5 \pm 1.7[1-8]$

Treatment duration (days)

$112.2 \pm 24.3$ [72-148]

Number of Octagam courses

$2.8 \pm 1.0[1-5]$

Total Octagam dose administered (g)

$280.0 \pm 151.5[60-600]$

Posology (g/kg per course)

$1.5 \pm 0.6[0.45-2.50]$

Data are presented as the mean \pm standard deviation $(\mathrm{SD})$ and range in square brackets, unless indicated otherwise

$I V I G$ Intravenous immunoglobulin, ONLS overall neuropathy limitation scale

Table 2 Effect of baseline demographics and exposure to Octagam on treatment response

\begin{tabular}{llll}
\hline Baseline demographics and exposure to Octagam & Responders $(\boldsymbol{n}=\mathbf{1 0})$ & Non-responders $(\boldsymbol{n}=\mathbf{1 4})$ & $\boldsymbol{p}$ values \\
\hline Gender, $n(\%)$ & & & \\
Male & $5(50.0)$ & $12(85.7)$ & 0.06 \\
Female & $5(50.0)$ & $2(14.3)$ & \\
Age (years) & $62.5 \pm 10.5$ & $63.1 \pm 11.6$ & 0.88 \\
Disease onset (months) & $32.8 \pm 49.4$ & $65.4 \pm 113.2$ & 0.64 \\
Initial ONLS score & $4.2 \pm 1.7$ & $3.0 \pm 1.5$ & 0.047 \\
Treatment duration (days) & $115.0 \pm 26.8$ & $110.1 \pm 23.2$ & 0.52 \\
Number of courses & $3.0 \pm 0.9$ & $2.6 \pm 1.2$ & 0.18 \\
Total dose administered (g) & $335.5 \pm 180.6$ & $240.4 \pm 118.0$ & 0.20 \\
Posology (g/kg per course) & $1.67 \pm 0.41$ & $1.39 \pm 0.65$ & 0.33 \\
\hline
\end{tabular}

Data are presented as the mean $\pm \mathrm{SD}$, unless indicated otherwise

required to establish whether treatment will eventually be individualised to target specific underlying pathologies.

Initially, Tegeline ${ }^{\circledR}$, a sugar-stabilised IVIG (LFB Group, Paris, France), was the only IVIG approved (based on retrospective data) for the treatment of CIDP in France. However, off-label use of other IVIGs is common, owing to the publication of several meta-analyses and guidelines confirming the efficacy of all commercialised IVIGs [6]. Not all IVIG preparations are alike in terms of tolerability. While IVIG treatment is usually well tolerated, IVIG preparations stabilised with sucrose are well known to 


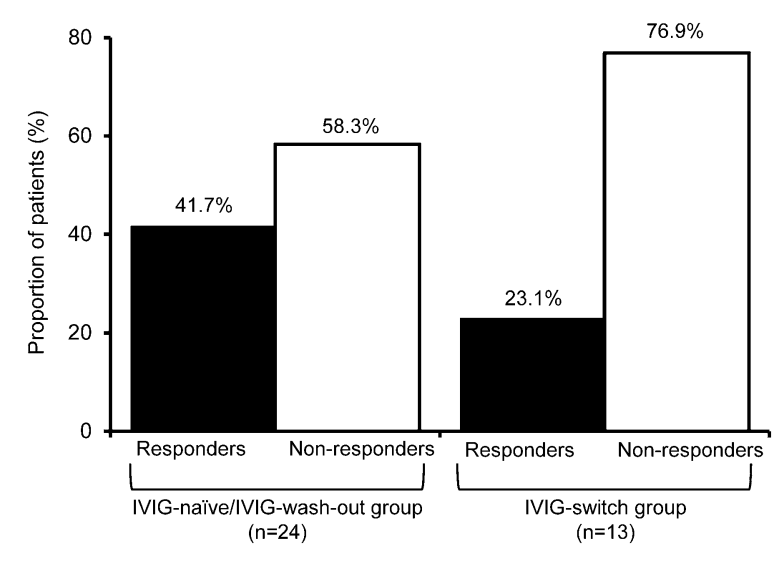

Fig. 1 Patient response to Octagam treatment at $4 \pm 1$ months after treatment initiation in the efficacy analysis population $(n=24)$. The Overall Neuropathy Limitation Scale score in responders decreased by $\geq 1$ point compared with baseline. The Intravenous immunoglobulin (IVIG)-naive group consisted of patients who had never received an IVIG before starting on Octagam treatment; IVIG-washout group consisted of patients who had stopped IVIG therapy $\geq 12$ weeks before starting Octagam treatment; IVIG-switch group consisted of patients who had replaced or stopped immunotherapy $<12$ weeks before starting Octagam treatment

be associated with acute renal failure, renal insufficiency and osmotic nephrosis [30]. The incidence of these renal adverse events is lower with IVIGs stabilised with non-sucrose sugars, such as glucose and maltose, and with D-sorbitol, mannitol, glycine and L-proline [30]. Octagam ${ }^{\circledR}$ (Octapharma AG, Lachen, Switzerland) is a sucrose-free liquid preparation of highly purified immunoglobulin G (IgG), which is often used off-label for CIDP as second-line treatment after Tegeline. The aim of this retrospective analysis was to describe the efficacy and safety of Octagam in CIDP patients in routine clinical practice in France.

\section{METHODS}

\section{Study Design and Data Collection}

This study was a retrospective analysis of the use of Octagam in a real-life setting at French medical centres specialising in the treatment of peripheral neuropathies. Centres provided data on consecutive patients fulfilling the following inclusion criteria: (1) legal adult age; (2) diagnosis of CIDP according to European Federation of Neurological Societies (EFNS) and the Peripheral Nerve Society (PNS) criteria [31]; and (3) initiation of therapy with Octagam $50 \mathrm{mg} /$ $\mathrm{mL}$ between 1999 and 2010. Exclusion criteria were: (1) presence of multifocal motor neuropathy; (2) presence of underlying systemic diseases, such as cancer, disseminated lupus erythematosus, human immunodeficiency virus infection, Gougerot-Sögren syndrome, and IgM-kappa paraprotein with or without antimyelin-associated-glycoprotein activity; and (3) introduction and modification of corticosteroid/immunosuppressive therapy during the 6-month period immediately prior to initiation of Octagam treatment.

\section{Treatment}

Octagam was administered according to routine clinical practice and based on the manufacturer's recommendations for injections. The posology of Octagam was recorded for all patients and for each course (total dose per course in grams and dose per course in grams per kilogram).

\section{Outcome Parameters}

The primary efficacy endpoint was the change in the Overall Neuropathy Limitation Scale (ONLS) score (range 0 [normal] to 12). The ONLS scale is used to evaluate the functional impairment of both the arms and legs [32]. A clinical response was defined as an improvement of disability status equivalent to a decrease of $\geq 1$ point in the ONLS score at $4 \pm 1$ months after the initiation of Octagam treatment compared with baseline; non-responders were patients with a stable or increased ONLS score at this same time point. During this period, treatment modification or initiation of a new treatment was not allowed. Patients were excluded from the efficacy analysis if: (1) the initial ONLS value was unknown or equal to zero; or (2) there was insufficient follow-up ( $<2$ months). Exposure to treatment 
was defined as the time between first Octagam administration and endpoint assessment at $4 \pm 1$ months.

\section{Safety Assessment}

For each patient, the laboratory results, including creatininaemia and creatinine clearance, were reported, when available. All adverse drug reactions occurring during the study period were routinely monitored and reported.

\section{Statistical Analyses and Sample Size}

Data on all patients included in the study were analysed using SAS software version 9.1 (SAS Institute, Cary, NC, USA). Statistical analyses were descriptive, and the 95\% confidence intervals (CI) were given when necessary. Patient characteristics are reported as a number and percentage for categorical variables and as mean \pm standard deviation (SD) and range for continuous variables. Quantitative variables were compared using twotailed Mann-Whitney $U$ tests for group comparisons. Categorical variables were compared using either the chi-square test or Fisher's exact test. ONLS scores before Octagam initiation and at the time of evaluation were compared using signed tests. All tests, if performed, were conducted with a bilateral approach at the 5\% threshold. For the analysis of ONLS development over time, missing values were replaced by the last observation carried forward method. Due to the retrospective nature of the data analysis and the rarity of the disease, no calculation of statistical sample size was performed.

The full analysis set included all patients who received at least one dose of Octagam and met the inclusion/exclusion criteria. After enrolment, patients were grouped according to previous IVIG exposure. Patients who had never received an IVIG before starting Octagam were included in the IVIG-naïve group; patients who had stopped IVIG therapy $\geq 12$ weeks before starting Octagam were included in the IVIGwashout group; and patients who had replaced or stopped immunotherapy $<12$ weeks before starting Octagam were included in the IVIGswitch group.
The efficacy analysis set included all patients in the IVIG-naïve or IVIG-washout groups, with data pooled according to methods used in the ICE study [23].

The safety analysis set consisted of all 47 patients included in the full analysis set and was not split into previous IVIG exposure groups.

\section{Compliance with Ethics Guidelines}

This study was approved by the CCTIRS (Comité Consultatif sur le Traitement de l'Information en matière de Recherche dans le domaine de la Santé) and the CNIL (Commission Nationale Informatique et Liberté) according to the legal requirements of a retrospective analysis in France. As such, written informed consent was not required.

\section{RESULTS}

\section{Baseline Characteristics of the Total Patient Population}

Between June and October 2010, medical records of 75 patients with CIDP treated with Octagam were identified and collected from 11 participating centres. Following an initial analysis of these records, 28 patients who did not meet the eligibility criteria were excluded, leaving 47 patients who were eligible for data collection.

Of the 47 patients included in the safety analysis set, $29(61.7 \%)$ were male. The mean age at diagnosis was $61.3 \pm 14.8$ (range 22-85 ) years. Fifteen patients were considered to be IVIG naïve; 14 patients were included in the IVIG-washout group; and 18 patients were included in the IVIG-switch group. Patients received a mean of $254.5 \pm 204.3$ days of Octagam treatment, corresponding to a mean of $5.2 \pm 2.7$ courses. The mean dose of Octagam received was $1.6 \pm 0.5 \mathrm{~g} / \mathrm{kg}$ per course.

\section{Baseline Characteristics of the Efficacy Analysis Set}

Of the 47 patients in the safety analysis set, 24 patients (11 IVIG-naïve and 13 IVIG-washout) 
met the inclusion criteria for the efficacy analysis (Table 1). Mean age at diagnosis was $59.2 \pm 14.9$ years (range: $22-79$ years) and mean duration of CIDP at start of Octagam treatment was $52.1 \pm 92.3$ months. Between baseline and endpoint assessment, the patients received on average $2.8 \pm 1.0$ courses of Octagam over a mean duration of $112 \pm 24$ days.

\section{Efficacy}

After $4 \pm 1$ months of Octagam treatment, mean ONLS scores significantly decreased in the IVIG-naïve and IVIG-washout group from $3.50 \pm 1.63$ at baseline to $3.08 \pm 1.73$ (Table 2). The mean ONLS change from baseline was $0.42 \pm 1.11 \quad(p=0.04)$, corresponding to a response rate of $41.7 \%$ (95\% CI 21.9-61.4; Fig. 1). Among the non-responders, 12 patients were stable and two patients (one patient each in the IVIG-naïve and IVIG-washout groups) showed worsening. Five IVIG-naïve patients responded to Octagam, a response rate of $45.5 \%$ (95\% CI 16.0-74.9), and five IVIG-washout patients were responders, a response rate of $38.5 \%$ (95\% CI 12.0-64.9).

There was no difference in age, number of IVIG infusions and delay between treatment onset and evaluation between the responders and non-responders. In contrast, the duration of CIDP before enrolment tended to be higher in non-responders, but the difference was not statistically significant (32.8 vs. 65.4 months in non-responders; $p=0.64$ ). The ONLS score at baseline was marginally higher in responders than non-responders $(4.2 \pm 1.7$ vs. $3.0 \pm 1.5$; $p=0.047)$. Furthermore, the mean posology and total dose received from baseline to study end were higher in responders, but this difference was not statistically significant (mean total dose of 335.5 vs. $240.4 \mathrm{~g}[p=0.20]$ and mean dosage of 1.7 vs. $1.4 \mathrm{~g} / \mathrm{kg}$ per treatment course $[p=0.33])$.

\section{Safety}

A total of 246 courses of Octagam were administered. Overall, $19(40.4 \%)$ of 47 patients reported 45 AEs (Table 3 ). The most frequently reported AEs per infusion were those affecting the nervous system $(24.4 \%$ of AEs, $17.0 \%$ of patients). Headaches were frequent $(20.0 \%$ of AEs, $14.9 \%$ of patients). Local reactions at the injection site (three cases in two patients) and asthenia (four cases in three patients) were the most frequently reported adverse reactions among the general disorders. Skin reactions (three cases, including lymphangitis in two patients and urticaria in one patient) and arthralgia-type pain (one case) were also reported. Two serious AEs considered by the physician as not being related to Octagam were reported for the same patient (appendectomy and peritonitis). No patient withdrew from treatment due to Octagam-related AEs. Nine AEs were considered to be moderate to severe in intensity and affected $10.6 \%$ of patients. Ten AEs were possibly or probably related to Octagam; these adverse reactions are already listed in the Summary of Product Characteristics and were reported by six patients for a total of seven different IVIG courses. The global incidence of AEs related to Octagam was 0.04 events/course $(10 / 246)$ or 0.13 events/patient (6/47). No clinically significant alteration in renal function was observed.

\section{DISCUSSION}

The results of this study show that Octagam is an effective treatment for patients with CIDP. Of the IVIG-naïve and IVIG-washout patients, $41.7 \%$ had improved their functional status by $4 \pm 1$ months post-initiation of Octagam treatment, as assessed by ONLS scores. AEs probably related to Octagam occurred in $12.8 \%$ of patients; none of these AEs were serious. The incidence of AEs related to Octagam was $4.1 \%$ per course.

Patients were included consecutively in this analysis, without any selection, reflecting the reallife management of patients treated with Octagam for CIDP in France. Our efficacy results are consistent with those from other studies on other IVIG brands used to treat CIDP [33-35]. Furthermore, the efficacy of IVIG in patients with CIDP has been shown in a recent Cochrane review that analysed the results of five randomised controlled trials involving 235 patients and comparing 
Table 3 Safety of Octagam

\begin{tabular}{ll}
\hline Safety parameters & Values $(\boldsymbol{n}=\mathbf{4} 7$ patients $)$ \\
\hline Total number of AEs reported & 45 \\
Headache & 9 \\
Systemic symptoms $^{\mathrm{b}}$ & 8 \\
Skin reactions $^{\mathrm{c}}$ & 3 \\
High blood pressure & 1 \\
Other & 24 \\
Number of IVIG courses & 246 \\
Number of courses with at least one AE probably or possibly related to Octagam, $n(\%)^{\mathrm{d}}$ & $7(2.8)$ \\
Number of patients with at least one AE, $n$ (\%) & $19(40.4)$ \\
Number of patients with at least one SAE, $n$ (\%) & $1^{\text {a }}(2.1)$ \\
Number of patients with at least one severe AE, $n$ (\%) & $1^{\text {a }}(2.1)$ \\
Number of patients with AE probably or possibly related to Octagam, $n(\%)$ & $6(12.8)$ \\
\hline
\end{tabular}

$A E$ Adverse event, $S A E$ serious adverse event

a Unrelated to treatment

b Systemic symptoms included asthenia $(n=4$ in 3 patients), chills $(n=1)$, nausea $(\mathrm{n}=1)$, mental confusion $(n=1)$ and drowsiness $(n=1)$

c Skin reactions included lymphangitis $(n=2$ in 2 patients) and urticaria $(n=1)$

d One course was associated with 2 AEs and another one with 3 AEs; the other 5 courses were associated with 1 AE.

various IVIG brands against placebo [12]. In that review, IVIG was found to be effective in improving disability for at least 2-6 weeks compared with placebo [12]. However, the clinical relevance of these results is open to discussion since different scores and response criteria were used in each trial. To avoid this evaluation bias, we evaluated our patients using the standardised and widely used ONLS score.

While we observed no correlation between age and rate of response, the time since CIDP onset tended to be longer in non-responders, although the difference was not statistically significant. This result may be due to the lack of power in the study. However, this finding suggests that the time since disease onset at treatment initiation is a potential predictive factor for non-response. Moreover, mean dose and the total dose of Octagam were higher in responders than in non-responders (not statistically significant). Thus, no relationship between dose and efficacy could be established. Of note, the mean dose reported in this study for the responders
$(1.67 \mathrm{~g} / \mathrm{kg})$ was closer to that recommended in the EFNS/PNS guidelines $(2.0 \mathrm{~g} / \mathrm{kg})$, compared to the mean dose for the non-responders $(1.39 \mathrm{~g} / \mathrm{kg})$ [31].

In this analysis, the safety profile of Octagam was unsurpassed compared to other IVIG preparations, with a very low incidence of AEs related to Octagam per course. These data are in accordance with results from previous doubleblind studies suggesting that IVIG safety is similar in everyday clinical practice and in randomised studies [29]. These findings are also consistent with those of a 10 -year prospective observational study investigating the safety of Octagam in primary and secondary immunodeficiencies and autoimmune diseases [36]. Furthermore, a randomised study investigating IVIG versus intravenous methylprednisolone in patients with CIDP showed that IVIG treatment was less frequently discontinued because of inefficacy, AEs or intolerance [29]. These data suggest that long-term IVIG therapy is better tolerated than corticosteroids, for which long- 
term tolerability is generally recognised as poor in the elderly and in those who present with concomitant medical conditions, such as hypertension, diabetes, obesity, peptic ulcer disease and osteoporosis [37]. Potential serious AEs commonly develop after as little as 2 months of exposure to corticosteroid.

The present study has certain limitations, such as the retrospective data collection and the small sample size. However, our findings are consistent with published data demonstrating the benefits and the safety of all IVIGs in patients with CIDP [33-35]. Future studies investigating adequate dosing and duration to achieve complete responses in the long-term use of IVIG and identifying different patterns of IVIG response in CIDP patients are warranted. One such study (ProCID; ClinicalTrials.gov identifier NCT02638207) assessing the efficacy and safety of three different doses of IVIG is currently ongoing, and the results are eagerly awaited.

\section{CONCLUSION}

These real-life results are in line with the efficacy and safety data of IVIG from randomised controlled studies. Several issues remain to be addressed and should become the focus of future studies, including the identification of distinctive patterns of IVIG response in CIDP patients. Identifying and characterising CIDP subtypes and their respective long-term response patterns would improve the effectiveness of the management of CIDP.

\section{ACKNOWLEDGEMENTS}

Funding. This study was funded by Octapharma, France SAS. The funder of the study was responsible for the design of the study, data extraction, data analysis and statistical analysis and contributed to data interpretation and writing of the manuscript. $\mathrm{CB}$ had final responsibility for the data provided. The article processing charges for this article were also funded by Octapharma.
Medical Writing, Editorial, and other Assistance. The authors would like to thank Jean-Charles Crave of Octapharma France SAS for the management of the study, Frédérick El Kouri for technical editing of the manuscript and Pierre Clerson of Soladis Clinical Studies for the statistical analysis of data. The authors would also like to thank Simone Boniface of Springer Healthcare Communications who provided English and technical editing and Stefan Wietek, a consultant for Octapharma, for critical review of the manuscript. This assistance was funded by Octapharma.

Authorship. All named authors meet the International Committee of Medical Journal Editors (ICMJE) criteria for authorship for this manuscript, take responsibility for the integrity of the work as a whole, and have given final approval to the version to be published.

Disclosures. Chafke Belmokhtar is an employee of Octapharma France SAS. Philippe Corcia has a financial relationship with Octapharma, France SAS, based on study honoraria. Etienne Godet has a financial relationship with Octapharma, France SAS, based on study honoraria. Pierre Lozeron, David Adams, Jérôme Franques, Arnaud Lacour, Etienne Godet, Mathieu Bataille, Odile Dubourg, Gilles Angibaud, Emilien Delmont, Françoise Bouhour, Philippe Corcia and Jean Pouget have nothing to declare.

Compliance with Ethics Guidelines. This study was approved by the CCTIRS (Comite Consultatif sur le Traitement de l'Information en matière de Recherche dans le domaine de la Santé) and the authorisation of the CNIL (Commission Nationale Informatique et Liberté), according to the legal requirements of a retrospective analysis in France. As this study was a retrospective analysis, written informed consent was not required.

Data Availaility. Access to the database used for this retrospective analysis is available if necessary.

Open Access. This article is distributed under the terms of the Creative Commons 
Attribution-NonCommercial 4.0 International License (http://creativecommons.org/licenses/ by-nc/4.0/), which permits any noncommercial use, distribution, and reproduction in any medium, provided you give appropriate credit to the original author(s) and the source, provide a link to the Creative Commons license, and indicate if changes were made.

\section{REFERENCES}

1. Said G. Chronic inflammatory demyelinating polyneuropathy. Neuromuscul Disord. 2006;16(5):293-303. https://doi.org/10.1016/j. nmd.2006.02.008.

2. Lunn MP, Manji H, Choudhary PP, Hughes RA, Thomas PK. Chronic inflammatory demyelinating polyradiculoneuropathy: a prevalence study in South East England. J Neurol Neurosurg Psychiatry. 1999;66(5):677-80.

3. McLeod JG, Pollard JD, Macaskill P, Mohamed A, Spring P, Khurana V. Prevalence of chronic inflammatory demyelinating polyneuropathy in New South Wales. Australia. Ann Neurol. 1999;46(6):910-3.

4. Laughlin RS, Dyck PJ, Melton LJ 3rd, Leibson C, Ransom J. Incidence and prevalence of CIDP and the association of diabetes mellitus. Neurology. 2009;73(1):39-45. https://doi.org/10.1212/WNL. 0b013e3181aaea47.

5. Mygland A, Monstad P. Chronic polyneuropathies in Vest-Agder. Norway. Eur J Neurol. 2001;8(2):157-65.

6. Magy L, Vallat JM. Peripheral neuropathies. Rev Prat. 2009;59(6):839-40.

7. Barohn RJ, Kissel JT, Warmolts JR, Mendell JR. Chronic inflammatory demyelinating polyradiculoneuropathy. Clinical characteristics, course, and recommendations for diagnostic criteria. Arch Neurol. 1989;46(8):878-84.

8. van der Meché FG, Vermeulen $M$, Busch HF. Chronic inflammatory demyelinating polyneuropathy. Conduction failure before and during immunoglobulin or plasma therapy. Brain. 1989;112(Pt 6):1563-71.

9. van Doorn PA, Vermeulen M, Brand A, Mulder PG, Busch HF. Intravenous immunoglobulin treatment in patients with chronic inflammatory demyelinating polyneuropathy. Clinical and laboratory characteristics associated with improvement. Arch Neurol. 1991;48(2):217-20.

10. Koike H, Nishi R, Ikeda $\mathrm{S}$, et al. Ultrastructural mechanisms of macrophage-induced demyelination in CIDP. Neurology. 2018;91(23):1051-60. https://doi.org/10.1212/WNL.0000000000006625.

11. Koike H, Kadoya M, Kaida KI, et al. Paranodal dissection in chronic inflammatory demyelinating polyneuropathy with anti-neurofascin-155 and anti-contactin-1 antibodies. J Neurol Neurosurg Psychiatry. 2017;88(6):465-73. https://doi.org/10. 1136/jnnp-2016-314895.

12. Eftimov F, Winer JB, Vermeulen M, de Haan R, van Schaik IN. Intravenous immunoglobulin for chronic inflammatory demyelinating polyradiculoneuropathy. Cochrane Database Syst Rev. 2013;12: CD001797. https://doi.org/10.1002/ 14651858.CD001797.pub3.

13. Hughes RA, Mehndiratta MM, Rajabally YA. Corticosteroids for chronic inflammatory demyelinating polyradiculoneuropathy. Cochrane Database Syst Rev. 2017;11:CD002062. https://doi.org/10.1002/ 14651858.CD002062.pub4

14. Mehndiratta MM, Hughes RA, Pritchard J. Plasma exchange for chronic inflammatory demyelinating polyradiculoneuropathy. Cochrane Database Syst Rev. 2015;8:CD003906. https://doi.org/10.1002/ 14651858.CD003906.pub4.

15. Oaklander AL, Lunn MP, Hughes RA, van Schaik IN, Frost C, Chalk CH. Treatments for chronic inflammatory demyelinating polyradiculoneuropathy (CIDP): an overview of systematic reviews. Cochrane Database Syst Rev. 2017;1:CD010369. https://doi.org/10.1002/14651858.CD010369. pub2.

16. Faed JM, Day B, Pollock M, Taylor PK, Nukada H, Hammond-Tooke GD. High-dose intravenous human immunoglobulin in chronic inflammatory demyelinating polyneuropathy. Neurology. 1989;39(3):422-5.

17. van Doorn PA, Brand A, Strengers PF, Meulstee J, Vermeulen $M$. High-dose intravenous immunoglobulin treatment in chronic inflammatory demyelinating polyneuropathy: a doubleblind, placebo-controlled, crossover study. Neurology. 1990;40(2):209-12.

18. van Doorn PA, Rossi F, Brand A, van Lint M, Vermeulen M, Kazatchkine MD. On the mechanism of high-dose intravenous immunoglobulin treatment of patients with chronic inflammatory demyelinating polyneuropathy. J Neuroimmunol. 1990;29(1-3):57-64. 
19. Vermeulen M, van der Meché FG, Speelman JD, Weber A, Busch HF. Plasma and gamma-globulin infusion in chronic inflammatory polyneuropathy. J Neurol Sci. 1985;70(3):317-26.

20. Cornblath DR, Chaudhry V, Griffin JW. Treatment of chronic inflammatory demyelinating polyneuropathy with intravenous immunoglobulin. Ann Neurol. 1991;30(1):104-6. https://doi.org/10.1002/ ana.410300119.

21. Mendell JR, Barohn RJ, Freimer ML, et al. Randomized controlled trial of IVIg in untreated chronic inflammatory demyelinating polyradiculoneuropathy. Neurology. 2001;56(4):445-9.

22. Kuitwaard K, Fokkink WR, Brusse E, et al. Maintenance IV immunoglobulin treatment in chronic inflammatory demyelinating polyradiculoneuropathy. J Peripher Nerv Syst. 2017;22(4):425-32. https://doi.org/10.1111/jns.12242.

23. Hughes RA, Donofrio P, Bril V, et al. Intravenous immune globulin (10\% caprylate-chromatography purified) for the treatment of chronic inflammatory demyelinating polyradiculoneuropathy (ICE study): a randomised placebo-controlled trial. Lancet Neurol. 2008;7(2):136-44. https://doi.org/10. 1016/S1474-4422(07)70329-0.

24. Kuwabara S, Mori M, Misawa S, et al. Intravenous immunoglobulin for maintenance treatment of chronic inflammatory demyelinating polyneuropathy: a multicentre, open-label, 52-week phase III trial. J Neurol Neurosurg Psychiatry. 2017;88(10):832-8. https://doi.org/10.1136/jnnp2017-316427.

25. Querol L, Rojas-Garcia R, Casasnovas C, et al. Longterm outcome in chronic inflammatory demyelinating polyneuropathy patients treated with intravenous immunoglobulin: a retrospective study. Muscle Nerve. 2013;48(6):870-6. https://doi.org/ $10.1002 /$ mus.23843.

26. Dyck PJ, Litchy WJ, Kratz KM, et al. A plasma exchange versus immune globulin infusion trial in chronic inflammatory demyelinating polyradiculoneuropathy. Ann Neurol. 1994;36(6):838-45. https://doi.org/10.1002/ana.410360607.

27. Hughes R, Bensa S, Willison H, et al. Randomized controlled trial of intravenous immunoglobulin versus oral prednisolone in chronic inflammatory demyelinating polyradiculoneuropathy. Ann Neurol. 2001;50(2):195-201.

28. Gaebel K, Blackhouse G, Campbell K, et al. Intravenous immunoglobulin for the treatment of chronic inflammatory demyelinating polyradiculoneuropathy: a systematic review and meta-analysis. Open Med. 2010;4(3):e154-e166166.
29. Nobile-Orazio E, Cocito D, Jann S, et al. Intravenous immunoglobulin versus intravenous methylprednisolone for chronic inflammatory demyelinating polyradiculoneuropathy: a randomised controlled trial. Lancet Neurol. 2012;11(6):493-502. https:// doi.org/10.1016/S1474-4422(12)70093-5.

30. Dantal J. Intravenous immunoglobulins: in-depth review of excipients and acute kidney injury risk. Am J Nephrol. 2013;38(4):275-84. https://doi.org/ $10.1159 / 000354893$.

31. European Federation of Neurological Societies/Peripheral Nerve Society. European Federation of Neurological Societies/Peripheral Nerve Society Guideline on management of paraproteinemic demyelinating neuropathies. Report of a Joint Task Force of the European Federation of Neurological Societies and the Peripheral Nerve Society-first revision. J Peripher Nerv Syst. 2010;15(3):185-95. https://doi.org/10.1111/j.1529-8027.2010.00278.x.

32. Graham RC, Hughes RA. A modified peripheral neuropathy scale: the overall neuropathy limitations scale. J Neurol Neurosurg Psychiatry. 2006;77(8):973-6. https://doi.org/10.1136/jnnp. 2005.081547 .

33. Léger JM, Chassande B, Musset L, Meininger V, Bouche P, Baumann N. Intravenous immunoglobulin therapy in multifocal motor neuropathy: a double-blind, placebo-controlled study. Brain. 2001;124(Pt 1):145-53.

34. Vermeulen M, van Doorn PA, Brand A, Strengers PF, Jennekens FG, Busch HF. Intravenous immunoglobulin treatment in patients with chronic inflammatory demyelinating polyneuropathy: a double blind, placebo controlled study. J Neurol Neurosurg Psychiatry. 1993;56(1):36-9.

35. Latov N, Deng C, Dalakas MC, et al. Timing and course of clinical response to intravenous immunoglobulin in chronic inflammatory demyelinating polyradiculoneuropathy. Arch Neurol. 2010;67(7):802-7. https://doi.org/10.1001/ archneurol.2010.105.

36. Debes A, Bauer M, Kremer S. Tolerability and safety of the intravenous immunoglobulin octagam: a 10-year prospective observational study. Pharmacoepidemiol Drug Saf. 2007;16(9):1038-47. https:// doi.org/10.1002/pds.1449.

37. Gorson KC. An update on the management of chronic inflammatory demyelinating polyneuropathy. Ther Adv Neurol Disord. 2012;5(6):359-73. https://doi.org/10.1177/ 1756285612457215 . 